\title{
New Aspects in Obesity and Diabetes: Involvement in Regucalcin, a Suppressor Protein of Cell Signaling
}

\section{Masayoshi Yamaguchi*}

Department of Hematology and Medical Oncology, Emory University School of Medicine, Atlanta, USA

Obesity and diabetes (type 1 and 2 diabetes) are currently a major health problem worldwide with growing in prevalence. The incidence of metabolic disease, including type 2 diabetes with obesity, has increased to epidemic levels. Obesity and diabetes induce secondary diseases with various pathophysiologic states, which are important in clinical aspects including cardiovascular disease, neural disturbance, kidney disease, osteoporosis and cancer [1-6]. Obesity is based on stimulation of adipogenesis. Bone marrow mesenchymal stem cells are multipotent cells, which among other cell lineages, and give to differentiate into adipocytes, osteoblasts, chondrocytes and myoblasts [1]. This occurs through cross talk between complex signaling pathways including those derived from bone morphogenic proteins, winglesstype MMTV integration site (Wnt) proteins, hedgehogs, delta/ jagged proteins, fibroblastic growth factors, insulin, insulin-like growth factors, and transcriptional regulators of adipocyte and osteoblast differentiation including peroxisome proliferators-activated receptorgamma $(P P A R \gamma)$ and runt-related transcription factor 2 (Runx2) [13]. Insulin, which is secreted by feeding, stimulates adipogenesis from bone marrow mesenchymal stem cells. In addition, bone marrow adiposity and mature adipocytes with obesity greatly produces tumor necrosis factor- $\alpha$ (TNF- $\alpha$ ), an inflammatory cytokine [4]. This TNF- $\alpha$ may cause insulin resistance that leads to type 2 diabetes.

Various hormones and cytokines, which include leptin, adiponectin, insulin, epinephrine, cortisol, glucagon, TNF- $\alpha$ and other factors, are well known as key molecules that relate to obesity and diabetes. Disturbance of these factors may play an important role in pathophysiologic conditions of obesity and diabetes. In addition, it has been proposed that regucalcin, a suppressor protein of intracellular signaling systems [7], may be a key molecule in obesity, diabetes and osteoporosis. Regucalcin has been demonstrated to stimulate adipogenesis in mouse bone marrow cell culture in vitro [8], suggesting an involvement as a stimulatory factor in adipogenesis.

Regucalcin, which was discovered in 1978 [9], plays a multifunctional role as a suppressor protein in signal transduction in various cell types and plays a cell physiologic role in maintaining cell homeostasis for various stimuli [10-14]. Cytoplasmic regucalcin localizes into the nucleus, and it suppresses nuclear protein kinase and protein phosphatase activities and DNA and RNA synthesis and regulates gene expression for various proteins [15]. Regucalcin has also been shown to suppress protein synthesis and activate proteolysis, suggesting a role as suppressor in protein turnover [11]. Moreover, overexpression of endogenous regucalcin has been demonstrated to suppress cell proliferation and apoptosis induced through multisignaling pathways in various cell types $[16,17]$. Thus, regucalcin plays a pivotal role in cell regulation.

Moreover, there is growing evidence that regucalcin plays an important role in the regulation of glucose and lipid metabolism. Fasting-induced decrease in the hepatic regucalcin mRNA expression has been shown to restore after re-feeding in rats in vivo [18], suggesting that feeding is a physiologic factor in the regulation of the regucalcin gene expression. In addition, oral administration of glucose to fasted rats causes a significant increase in hepatic regucalcin
mRNA expression [18], suggesting an involvement of insulin secreted from pancreatic cells after glucose administration. Moreover, hepatic regucalcin mRNA expression is clearly elevated after a single subcutaneous administration of insulin to fasted rats in vivo [18]. In fact, insulin has been demonstrated to directly stimulate regucalcin mRNA and protein expressions in human hepatoma cells (HepG2) in vitro [19]. Thus, insulin, which is related to metabolism of blood glucose after feeding, stimulates regucalcin expression in liver cells. In addition, hepatic regucalcin expression has been shown to markedly decrease after a single subcutaneous administration of streptozotocin that induces type 1 diabetes [20]. These findings may support the view that regucalcin may be involved in liver metabolic disorder related to diabetes.

Deficiency of regucalcin has been reported to cause an impairment of glucose tolerance in regucalcin knockout (KO) mice [21]. Regucalcin $\mathrm{KO}$ mice causes a significant increase in blood glucose concentration and a decrease in serum insulin levels after glucose administration compared with wild-type mice in vivo [22], suggesting that regucalcin participates in the regulation of glucose metabolism related to insulin action. Insulin resistance may be modeled in culture system by using cloned rat hepatoma H4-II-E cells cultured with insulin and TNF-a in vitro [22]. This in vitro model nicely mimics insulin resistance in human type 2 diabetic mellitus. When H4-II-E cells are cultured in the presence of TNF- $\alpha$ plus insulin in vitro, regucalcin is identified as an important protein, which is involved in insulin resistance, by proteome analysis [23]. Thus, regucalcin may be a key molecule that is related to insulin resistance. Moreover, regucalcin has been demonstrated to stimulate glucose utilization and lipid production in H4-II-E cells in vitro [24]. Overexpression of endogenous regucalcin is found to stimulate the production of triglyceride and free fatty acid in H4-II-E cells cultured with or without the supplementation of glucose in the absence of insulin [24]. Regucalcin may stimulate lipid production that is linked to glucose metabolism in liver cells in vitro. Moreover, the effect of insulin, which enhances medium glucose consumption, triglyceride and free fatty acid productions in liver cells cultured with glucose supplementation, is suppressed by overexpression of regucalcin in vitro [25].

Molecular mechanism by which regucalcin regulates glucose

*Corresponding author: Masayoshi Yamaguchi, Ph.D., Department of Hematology and Medical Oncology, Emory University School of Medicine, Room C5054, 1365 C Clifton Road NE, Atlanta, GA 30322, USA, Tel: 404-664-7422; E-mail: yamamasa1155@yahoo.co.jp

Received January 03, 2014; Accepted January 08, 2014; Published January 13 2014

Citation: Yamaguchi M (2014) New Aspects in Obesity and Diabetes: Involvement in Regucalcin, a Suppressor Protein of Cell Signaling. J Mol Genet Med S1: 010. doi: 10.4172/1747-0862.S1-010

Copyright: (C) 2014 Yamaguchi M. This is an open-access article distributed under the terms of the Creative Commons Attribution License, which permits unrestricted use, distribution, and reproduction in any medium, provided the original author and source are credited 
metabolism related insulin action has been elucidated. Overexpression of regucalcin does not reveal stimulatory effects on the gene expression of enzymes, which are related to glucose and lipid metabolism, including acetyl-CoA carboxylase, HMG-CoA reductase, glucokinase and pyruvate kinase in liver cells after culture with or without glucose supplementation in the presence of insulin [23], although it is possible that regucalcin has a regulatory effect on various enzyme activities, which are related to glucose and lipid metabolism in liver cells. Interestingly, overexpression of regucalcin has been shown to have suppressive effects on the expression of rat insulin receptor (Insr) or phosphatidylinositol 3-kinase (PI3K) mRNAs enhanced after culture with glucose supplementation in the presence of insulin [23,24]. Suppressive effects of regucalcin on the expression of Insr and PI3K mRNAs may play an important role in insulin resistance in liver cells overexpressing endogenous regucalcin. Insulin resistance in the liver is associated with the pathogenesis of nonalcoholic fatty liver disease (NFLD), suggesting an involvement in lipid metabolic disorder.

Hepatocytes, which are obtained from regucalcin $\mathrm{KO}$ mice at 12 months of age, have been shown to contain many lipid droplets, abnormally enlarged mitochondria with indistinct cristae, and enlarged lysosomes filled with electron-dense bodies in the electron microscope as compared with that of wild-type mice [25]. Hepatic neutral lipids, total phospholipids, total triglyceride and cholesterol in regucalcin $\mathrm{KO}$ mice are found to markedly increase than those from age-matched wild-type mice [25]. Deficiency of regucalcin leads to accumulation of liver lipid components.

Moreover, regucalcin transgenic (TG) rats with overexpression of endogenous regucalcin have been shown to induce a remarkable of bone loss associated with increase in serum triglyceride and high-density lipoprotein (HDL)-cholesterol concentrations at the age of 36 weeks in vivo [22]. Serum free fatty acid, triglyceride, cholesterol or HDLcholesterol concentrations are markedly increased in regucalcin TG male and female rats at 14-50 weeks of age [26]. Thus, hyperlipidemia is uniquely induced in regucalcin TG rats with increasing age. The change in lipid components in the adipose and liver tissues of regucalcin TG rats with increasing age has also been shown in vivo [27]. Regucalcin is expressed in the adipose tissues of normal rats [27]. Triglyceride content in the adipose tissues is increased in regucalcin TG rats with aging [27]. Liver triglyceride, total cholesterol, free fatty acid and glycogen contents are decreased in regucalcin TG rats. The expression of regucalcin in the liver tissues is enhanced in regucalcin TG rats [27]. Regucalcin has been shown to have suppressive effects on the activations of glycogen particulate phosphorylase a, cytoplasmic pyruvate kinase, and fructose 1,6-diphosphatase in rat liver $[8,13]$. Regucalcin may suppress glycogen synthesis in the liver and stimulate glycogenolysis in regucalcin TG rats. As the result, lipid synthesis may be stimulated in the liver tissues of the TG rats in vivo. Leptin and adiponectine are adipokines that are involved in lipid metabolism [28]. Leptin mRNA expression in the adipose or liver tissues has been found to decrease in regucalcin TG rats with aging [27]. Adiponectin mRNA expression is not changed in the adipose tissues of the TG rats, while its level is decreased in the liver tissues [27]. These decreases may be partly involved in hyperlipidemia induced in regucalcin TG rats. Thus, regucalcin may play an important role in the disorder of lipid metabolism in the liver.

Hyperlipidemia has been shown to induce in the lipoprotein lipasedeficient mice [28], low-density lipoprotein (LDL) receptor-deficient mice [29], apolipoprotein C3-KO mice [30], apolipoprotein C1 TG mice [31], very LDL lipoprotein receptor KO mice [32], cholesterol
7 alpha-hydroxylase-deficient mice [33], apoE-deficient mice [34], and hepatic myr-Akt overexpressing mice [35]. These animal models for hyperlipidemia are involved in molecules that regulate lipid metabolism. Regucalcin has also been proposed to be a key molecule that regulates lipid metabolism.

As described above, regucalcin plays a physiological role in lipid and glucose metabolism. Regucalcin, which is stimulated by insulin, is identified as a molecule that is related to insulin resistance in liver cells. Deficiency of regucalcin impairs glucose tolerance and induces lipid accumulation in the liver of mice in vivo. Overexpression of regucalcin stimulates glycolysis and lipid production in the liver tissues of rats in vivo. Disturbance of hepatic regucalcin expression may leads to NFLD. Moreover, hyperlipidemia is induced in regucalcin TG rats in vivo. Thus, regucalcin may be a key molecule in lipid metabolic disorder and diabetes. Regucalcin may be a target molecule for therapy of these diseases. Development of further study will be expected.

\section{Author Disclosures}

The author has no conflicts of interest.

\section{Acknowledgements}

Regucalcin studies of the author was supported by a Grant-in-Aid for Scientific Research (C) No.63571053, No.02671006, No.04671362, No.06672193, No.08672522, No.10672048, No.13672292 and No.17590063 from the Ministry of Education, Science, Sports, and Culture, Japan. Also, the author was awarded from the Bounty of Encouragement Foundation in Pharmaceutical Research and the Bounty of the Yamanouchi Foundation for Research on Metabolic Disorders. This study was also supported by the Foundation for Biomedical Research on Regucalcin.

\section{References}

1. Minguell JJ, Erices A, Conget P (2001) Mesenchymal stem cells. Exp Biol Med (Maywood) 226: 507-520.

2. Muruganandan S, Roman AA, Sinal CJ (2009) Adipocyte differentiation of bone marrow-derived mesenchymal stem cells: cross talk with the osteoblastogenic program. Cell Mol Life Sci 66: 236-253.

3. Laudes M (2011) Role of WNT signalling in the determination of human mesenchymal stem cells into preadipocytes. J Mol Endocrinol 46: R65-R72.

4. Gharibi B, Abraham AA, Ham J, Evans BA (2011) Adenosine receptor subtype expression and activation influence the differentiation of mesenchymal stem cells to osteoblasts and adipocytes. J Bone Miner Res 26: 2112-2124.

5. Kawai M, Rosen CJ (2010) PPAR $\left.\right|^{3}$ : a circadian transcription factor in adipogenesis and osteogenesis. Nat Rev Endocrinol 6: 629-636.

6. Rosen CJ, Bouxsein ML (2006) Mechanisms of disease: is osteoporosis the obesity of bone? Nat Clin Pract Rheumatol 2: 35-43.

7. Yamaguchi M (2010) Regucalcin and metabolic disorders: osteoporosis and hyperlipidemia are induced in regucalcin transgenic rats. Mol Cell Biochem 341: 119-133.

8. Yamaguchi M, Weitzmann MN, Baile CA, Murata T (2012) Exogenous regucalcin suppresses osteoblastogenesis and stimulates adipogenesis in mouse bone marrow culture. Integr Biol (Camb) 4: 1215-1222.

9. Yamaguchi $\mathrm{M}$ (1992) A novel $\mathrm{Ca}^{2+}$-binding protein regucalcin and calcium inhibition. Regulatory role in liver cell function. In: Kohama K (Ed) Calcium inhibition: a new mode for $\mathrm{Ca}^{2+}$ regulation. Japan Sci Soc Press, Tokyo.

10. Shimokawa N, Yamaguchi M (1993) Molecular cloning and sequencing of the cDNA coding for a calcium-binding protein regucalcin from rat liver. FEBS Let 327: $251-255$

11. Yamaguchi M (2011) The transcriptional regulation of regucalcin gene expression. Mol Cell Biochem 346: 147-171.

12. Yamaguchi M (2000) Role of regucalcin in calcium signaling. Life Sci 66: 1769 1780.

13. Yamaguchi M (2005) Role of regucalcin in maintaining cell homeostasis and function (review). Int J Mol Med 15: 371-389. 
Citation: Yamaguchi M (2014) New Aspects in Obesity and Diabetes: Involvement in Regucalcin, a Suppressor Protein of Cell Signaling. J Mol Genet Med S1: 010. doi: 10.4172/1747-0862.S1-010

14. Yamaguchi M (2011) Regucalcin and cell regulation: role as a suppressor protein in signal transduction. Mol Cell Biochem 353: 101-137.

15. Yamaguchi M (2013) Role of regucalcin in cell nuclear regulation: involvement as a transcription factor. Cell Tissue Res 354: 331-341.

16. Yamaguchi M (2013) Suppressive role of regucalcin in liver cell proliferation: involvement in carcinogenesis. Cell Prolif 46: 243-253.

17. Yamaguchi M (2013) The anti-apoptotic effect of regucalcin is mediated through multisignaling pathways. Apoptosis 18: 1145-1153.

18. Yamaguchi M, Oishi K, Isogai M (1995) Expression of hepatic calcium-binding protein regucalcin mRNA is elevated by refeeding of fasted rats: involvement of glucose, insulin and calcium as stimulating factors. Mol Cell Biochem 142 $35-41$.

19. Murata T, Shinya N, Yamaguchi M (1997) Expression of calcium-binding protein regucalcin mRNA in the cloned human hepatoma cells (HepG2): stimulation by insulin. Mol Cell Biochem 175: 163-168.

20. Isogai M, Kurota H, Yamaguchi M (1997) Hepatic calcium-binding protein regucalcin concentration is decreased by streptozotocin-diabetic state and ethanol ingestion in rats. Mol Cell Biochem 168: 67-72.

21. Hasegawa G, Yamasaki M, Kadono M, Tanaka M, Asano M, et al. (2010) Senescence marker protein-30/gluconolactonase deletion worsens glucose tolerance through impairment of acute insulin secretion. Endocrinology 151: 529-536.

22. Solomon SS, Buss N, Shull J, Monnier S, Majumdar G, et al. (2005) Proteome of $\mathrm{H}-411 \mathrm{E}$ (liver) cells exposed to insulin and tumor necrosis factor-alpha: analysis of proteins involved in insulin resistance. J Lab Clin Med 145: 275-283.

23. Nakashima C, Yamaguchi M (2006) Overexpression of regucalcin enhances glucose utilization and lipid production in cloned rat hepatoma H4-II-E cells: Involvement of insulin resistance. J Cell Biochem 99: 1582-1592.

24. Nakashima C, Yamaguchi M (2007) Overexpression of regucalcin suppresses gene expression of insulin signaling-related proteins in cloned rat hepatoma H4-II-E cells: involvement of insulin resistance. Int J Mol Med 20: 709-716.

25. Ishigami A, Kondo Y, Nanba R, Ohsawa T, Handa S, et al. (2004) SMP30 deficiency in mice causes an accumulation of neutral lipids and phospholipids in the liver and shortens the life span. Biochem Biophys Res Commun 315 575-580.
26. Yamaguchi M, Igarashi A, Uchiyama S, Sawada N (2004) Hyperlipidemia is induced in regucalcin transgenic rats with increasing age. Int $\mathrm{J}$ Mol Med 14: 647-651.

27. Yamaguchi M, Nakagawa T (2007) Change in lipid components in the adipose and liver tissues of regucalcin transgenic rats with increasing age: suppression of leptin and adiponectin gene expression. Int J Mol Med 20: 323-328.

28. Ronti T, Lupattelli G, Mannarino E (2006) The endocrine function of adipose tissue: an update. Clin Endocrinol (Oxf) 64: 355-365.

29. Weinstock $\mathrm{PH}$, Bisgaier $\mathrm{CL}$, Aalto-Setälä $\mathrm{K}$, Radner $\mathrm{H}$, Ramakrishnan $\mathrm{R}$, et al. (1995) Severe hypertriglyceridemia, reduced high density lipoprotein, and neonatal death in lipoprotein lipase knockout mice. Mild hypertriglyceridemia with impaired very low density lipoprotein clearance in heterozygotes. $\mathrm{J}$ Clin Invest 96: 2555-2568.

30. Lichtman AH, Clinton SK, liyama K, Connelly PW, Libby P, et al. (1999) Hyperlipidemia and atherosclerotic lesion development in LDL receptor-deficien mice fed defined semipurified diets with and without cholate. Arterioscler Thromb Vasc Biol 19: 1938-1944.

31. Jong MC, Havekes LM (2000) Insights into apolipoprotein C metabolism from transgenic and gene-targeted mice. Int J Tissue React 22: 59-66.

32. Koopmans SJ, Jong MC, Que I, Dahlmans VE, Pijl H, et al. (2001) Hyperlipidaemia is associated with increased insulin-mediated glucose metabolism, reduced fatty acid metabolism and normal blood pressure in transgenic mice overexpressing human apolipoprotein C1. Diabetologia 44: 437-443.

33. Yagyu H, Lutz EP, Kako Y, Marks S, Hu Y, et al. (2002) Very low density lipoprotein (VLDL) receptor-deficient mice have reduced lipoprotein lipase activity. Possible causes of hypertriglyceridemia and reduced body mass with VLDL receptor deficiency. J Biol Chem 277: 10037-10043.

34. Chen JY, Levy-Wilson B, Goodart S, Cooper AD (2002) Mice expressing the human CYP7A1 gene in the mouse CYP7A1 knock-out background lack induction of CYP7A1 expression by cholesterol feeding and have increased hypercholesterolemia when fed a high fat diet. J Biol Chem 277: 42588-42595.

35. Fazio S, Linton MF (2001) Mouse models of hyperlipidemia and atherosclerosis. Front Biosci 6: D515-525.

36. Ono H, Shimano H, Katagiri H, Yahagi N, Sakoda H, et al. (2003) Hepatic Akt activation induces marked hypoglycemia, hepatomegaly, and hypertriglyceridemia with sterol regulatory element binding protein involvement. Diabetes 52 2905-2913. 